\title{
Editorial: Brown Adipose Tissue: From Heat Production in Rodents to Metabolic Health in Humans
}

\author{
Maria Chondronikola ${ }^{1,2 *}$, Alexander Bartelt ${ }^{3,4,5,6}$, Antonio Vidal-Puig ${ }^{7,8}$ \\ and Kirsi A. Virtanen ${ }^{9,10,11}$ \\ ${ }^{1}$ Department of Nutrition, University of California Davis, Davis, CA, United States, ${ }^{2}$ Department of Nutritional Sciences and \\ Dietetics, Harokopio University of Athens, Athens, Greece, ${ }^{3}$ Institute for Cardiovascular Prevention, Ludwig-Maximilians- \\ University, Munich, Germany, ${ }^{4}$ German Center for Cardiovascular Research, Partner Site Munich Heart Alliance, \\ Munich, Germany, ${ }^{5}$ Institute for Diabetes and Cancer, Helmholtz Center Munich, German Research Center for Environmental \\ Health, Neuherberg, Germany, ${ }^{6}$ Department of Molecular Metabolism, Harvard T.H. Chan School of Public Health, Boston, \\ MA, United States, 7 University of Cambridge Metabolic Research Laboratories, Institute of Metabolic Science, Medical \\ Research Council, Metabolic Diseases Unit, Cambridge, United Kingdom, ${ }^{8}$ Wellcome Trust Sanger Institute, Hinxton, \\ United Kingdom, ${ }^{9}$ Turku PET Centre, Turku University Hospital, Turku, Finland, ${ }^{10}$ Department of Public Health and Clinical \\ Nutrition, University of Eastern Finland, Kuopio, Finland, ${ }^{11}$ Department of Endocrinology and Clinical Nutrition, Kuopio \\ University Hospital, Kuopio, Finland
}

\section{OPEN ACCESS}

Edited and reviewed by: Cunming Duan, University of Michigan, United States

*Correspondence: Maria Chondronikola mchondr@ucdavis.edu

Specialty section: This article was submitted to Experimental Endocrinology,

a section of the journa

Frontiers in Endocrinology

Received: 09 July 2021 Accepted: 14 July 2021

Published: 26 July 2021

Citation:

Chondronikola M, Bartelt A, Vidal-Puig A and Virtanen KA (2021) Editorial: Brown Adipose Tissue:

From Heat Production in Rodents to Metabolic Health in Humans.

Front. Endocrinol. 12:739065. doi: 10.3389/fendo.2021.739065
Keywords: adipose tissue, brown, metabolism and obesity, thermogenesis, adipose tissue beigeing

Editorial on the Research Topic

Brown Adipose Tissue: From Heat Production in Rodents to Metabolic Health in Humans

\section{INTRODUCTION}

The unequivocal demonstration of functional brown adipose tissue (BAT) in adult humans (1-3) has led to a surge of scientific interest in its role in metabolic health. More recently, the discovery that white adipose tissue (WAT) can adopt a phenotype similar to BAT (a process also known as browning or beigeing of WAT) (4) has further fueled the hope to exploit the powers of thermogenic adipocytes against the obesity-related metabolic disorders. One of the unique characteristics of the thermogenic adipocytes is the large number of mitochondria enriched in uncoupling protein 1 (UCP1), which hotwires oxidative phosphorylation resulting in thermogenesis (5). Upon activation (in response to neuroendocrine, metabolic or dietary factors), thermogenic adipocytes increase their metabolic rate, oxygen consumption and accelerate glucose, lipid, and branched-chain amino acid metabolism (6). BAT has also been implicated as a potential endocrine organ, affecting the metabolic activity of distant tissues to coordinate whole-body metabolism (7). Although research efforts to this date have improved the current understanding of the regulation and metabolic significance of BAT and the thermogenic adipocytes, many questions remain to be answered. The manuscripts included in this Research Topic: i) summarize the current knowledge on the origins and plasticity of the thermogenic adipocytes and their potential role in metabolic health, ii) provide mechanistic insights on pathways implicated in thermogenesis, and iii) discuss current research gaps and how those can be addressed in the future. 


\section{MOLECULAR MECHANISMS FOR THE MAINTENANCE OF THE THERMOGENIC ADIPOCYTES}

In this Research Topic, Rabbie summarizes the current knowledge on the origins of thermogenic adipocytes (8). This review also provides a comprehensive overview of the transcriptional and epigenetic factors involved in their development and maintenance. The author emphasizes the need for future research to improve the current understanding of the mechanisms involved in the bidirectional transition between the thermogenic adipocytes and the classical white adipocytes.

\section{THE ROLE OF BAT IN HUMAN METABOLISM}

The role of BAT in metabolic health is currently a topic of major scientific interest and debate. Pan et al. summarize the differences between rodent and human BAT, the methods for the assessment of human BAT, and the pathways regulating BAT thermogenesis (Pan et al.). McNeill et al. review the current evidence on substrate utilization by human BAT. Advanced medical imaging techniques and microdialysis have been used to assess substrate utilization in BAT. BAT has high capacity for plasma glucose and free and dietary fatty acid uptake especially in response to cold, while BAT also utilizes intracellular triglycerides for thermogenesis. Additionally, intermediate metabolites (i.e., pyruvate and lactate) and glutamate may fuel BAT thermogenesis and/or play a role in the regulation of BAT. Limitations of the currently available methods hinder the efforts to further understand substrate utilization by BAT further.

\section{MECHANISTIC INSIGHTS IN THE REGULATION OF ADIPOSE TISSUE THERMOGENESIS}

\section{Electrical Neurostimulation (EN) for the Activation of BAT}

Although the role of the nervous system and adrenergic signaling in the regulation of BAT metabolism is well-established (5), the currently available $\beta$-adrenergic agonists lack tissue-specificity, limiting their clinical utility due to cardiovascular side effects (8). $\mathrm{Li}$ et al. reported that EN of BAT in rodents increased BAT thermogenesis and decreased its intracellular lipid content, whereas $\beta 3$-adrenergic blockade prevented the stimulation of BAT thermogenesis, suggesting that EN acts via beta3adrenergic signaling ( $\mathrm{Li}$ et al.). EN is a novel tissue-specific approach for the activation of BAT without the cardiovascular side effects of the systemic $\beta$-adrenergic stimulation.

\section{The Role of Macrophages in BAT Thermogenesis}

Although resident immune cells in BAT have been implicated in the regulation of BAT, their role remains debatable (9). Fischer et al. reported that thermoneutrality led to pronounced macrophage infiltration of BAT, while cold exposure had the opposite effect (Fischer et al.). Moreover, exposure to thermoneutrality or room temperature conditions before a cold challenge did not affect the cold-induced transcriptomic response in BAT suggesting that the presence of macrophages in BAT may not affect thermogenesis. Future investigations are needed to assess the role of macrophages in BAT function comprehensively.

\section{Calsyntenin3 $\beta$ Regulation in BAT}

Recent results from studies in rodents support that the neurotrophic and thermogenic calsyntenin $3 \beta-S 100 \mathrm{~b}$ axis regulates innervation in thermogenic adipose tissue (10). Plucinska et al. characterized the enrichment patterns of calsyntenin $3 \beta$ in various adipose tissue depots (Plucińska et al.). In humans, calsyntenin $3 \beta$ gene expression was higher in the perirenal multilocular BAT depot than the subcutaneous WAT, while its expression was positively associated with UCP1 expression. In rodents, calsyntenin $3 \beta$ gene expression was preferentially enriched in BAT and the enrichment pattern was sensitive to various physiological challenges (i.e., cold exposure, rewarming, and obesity). Calsyntenin $3 \beta$ is a promising target for the regulation of adipose-to-neuro axis.

\section{IN VITRO MODELS FOR THE STUDY OF HUMAN BAT}

To this date, studies in animal models have been the primary source of knowledge on the cellular identity, plasticity, metabolic regulation and significance of the thermogenic adipocytes. However, this classic experimental approach does not account for interspecies differences between humans and rodents. Considering the high cost and limitations of clinical research, it is essential to establish efficient preclinical methods for studying of the thermogenic adipocytes. Smartly designed in vitro experiments can be used in conjunction with in vivo studies to address the open questions in the thermogenic adipocyte biology. Samuelson and Vidal-Puig reviewed the current methods studying of human BAT in vitro including immortalizing primary human brown adipocytes, multipotent stem cells, and human pluripotent stem cells and the advantages and limitations of each method (Samuelson and Vidal-Puig). Optimization of the current in vitro models to more closely recapitulate the in vivo microenvironment of the thermogenic adipocytes (e.g., innervation, vascularization, extracellular matrix, presence of other cells, and tissue architecture) is of paramount importance to accelerate scientific progress in realizing the role of BAT in the context of the obesity-related metabolic complications.

\section{CONCLUSION}

BAT and thermogenic adipocytes constitute emerging targets against obesity and its related metabolic diseases that affect the well-being and quality of life of millions of people worldwide. To this date, the research progress on better understanding the origins, metabolic regulation and significance of BAT and the 
thermogenic adipocytes has been substantial. Nevertheless, many questions remain open. The most critical rate-limiting bottleneck hindering the scientific progress in the field of human BAT is the lack of tools to efficiently establish the signaling pathways regulating adipose tissue thermogenesis and to understand its metabolic significance at the tissue-specific and whole-body level. Answering those questions is critical to establish the thermogenic adipose tissue as a therapeutic target against metabolic disease.

\section{AUTHOR CONTRIBUTIONS}

$\mathrm{MC}$ and $\mathrm{KV}$ wrote the manuscript. AVP and $\mathrm{AB}$ edited the manuscript. All authors contributed to the article and approved the submitted version.

\section{REFERENCES}

1. Cypess AM, Lehman S, Williams G, Tal I, Rodman D, Goldfine AB, et al. Identification and Importance of Brown Adipose Tissue in Adult Humans. N Engl J Med (2009) 360:1509-17. doi: 10.1056/NEJMoa0810780

2. Virtanen KA, Lidell ME, Orava J, Heglind M, Westergren R, Niemi T, et al. Functional Brown Adipose Tissue in Healthy Adults. N Engl J Med (2009) 360:1518-25. doi: 10.1056/NEJMoa0808949

3. van Marken Lichtenbelt WD, Vanhommerig JW, Smulders NM, Drossaerts JM, Kemerink GJ, Bouvy ND, et al. Cold-Activated Brown Adipose Tissue in Healthy Men. N Engl J Med (2009) 360:1500-8. doi: 10.1056/NEJMoa0808718

4. Wu J, Bostrom P, Sparks LM, Ye L, Choi JH, Giang AH, et al. Beige Adipocytes are a Distinct Type of Thermogenic Fat Cell in Mouse and Human. Cell (2012) 150:366-76. doi: 10.1016/j.cell.2012.05.016

5. Cannon B, Nedergaard J. Brown Adipose Tissue: Function and Physiological Significance. Physiol Rev (2004) 84:277-359. doi: 10.1152/physrev.00015.2003

6. Chondronikola M, Sidossis LS. Brown and Beige Fat: From Molecules to Physiology. Biochim Biophys Acta Mol Cell Biol Lipids (2019) 1864:91-103. doi: 10.1016/j.bbalip.2018.05.014

7. Gavaldà-Navarro A, Villarroya J, Cereijo R, Giralt M, Villarroya F. The Endocrine Role of Brown Adipose Tissue: An Update on Actors and Actions. Rev Endocrine Metab Disord (2021). doi: 10.1007/s11154-021-09640-6

8. Chen KY, Brychta RJ, Abdul Sater Z, Cassimatis TM, Cero C, Fletcher LA, et al. Opportunities and Challenges in the Therapeutic Activation of Human Energy Expenditure and Thermogenesis to Manage Obesity. J Biol Chem (2020) 295:1926-42. doi: 10.1074/jbc.REV119.007363

\section{FUNDING}

MC is supported by the USDA National Institute of Food and Agriculture, Hatch project number CA-D-NTR-2618-H. $\mathrm{AB}$ was supported by the Deutsche Forschungsgemeinschaft Sonderforschungsbereich 1123 (B10), a Deutsches Zentrum für Herz-Kreislauf-Forschung Junior Research Group Grant, and the European Research Council Starting Grant PROTEOFIT.

\section{ACKNOWLEDGMENTS}

We are grateful to all the authors and reviewers for their contributions to this Research Topic.

9. Villarroya F, Cereijo R, Villarroya J, Gavaldà-Navarro A, Giralt M. Toward an Understanding of How Immune Cells Control Brown and Beige Adipobiology. Cell Metab (2018) 27:954-61. doi: 10.1016/j.cmet. 2018.04.006

10. Zeng X, Ye M, Resch JM, Jedrychowski MP, Hu B, Lowell BB, et al. Innervation of Thermogenic Adipose Tissue via a Calsyntenin 3ß-S100b Axis. Nature (2019) 569:229-35. doi: 10.1038/s41586-0191156-9

Conflict of Interest: The authors declare that the research was conducted in the absence of any commercial or financial relationships that could be construed as a potential conflict of interest.

Publisher's Note: All claims expressed in this article are solely those of the authors and do not necessarily represent those of their affiliated organizations, or those of the publisher, the editors and the reviewers. Any product that may be evaluated in this article, or claim that may be made by its manufacturer, is not guaranteed or endorsed by the publisher.

Copyright (c) 2021 Chondronikola, Bartelt, Vidal-Puig and Virtanen. This is an openaccess article distributed under the terms of the Creative Commons Attribution License (CC BY). The use, distribution or reproduction in other forums is permitted, provided the original author(s) and the copyright owner(s) are credited and that the original publication in this journal is cited, in accordance with accepted academic practice. No use, distribution or reproduction is permitted which does not comply with these terms. 